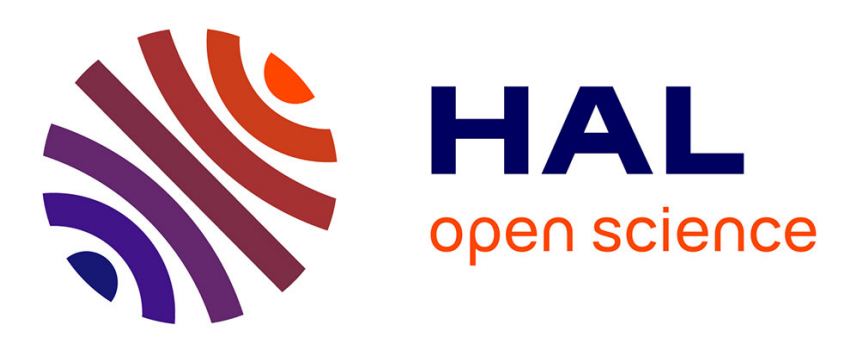

\title{
La gestion publique de la nature littorale, en quête de légitimité? L'exemple du Conservatoire du Littoral sur les rivages guyanais
}

Céline Barthon, Vincent Andreu-Boussut, Moise Tsayem Demaze

\section{To cite this version:}

Céline Barthon, Vincent Andreu-Boussut, Moise Tsayem Demaze. La gestion publique de la nature littorale, en quête de légitimité? L'exemple du Conservatoire du Littoral sur les rivages guyanais. 2009. halshs-00432789

\author{
HAL Id: halshs-00432789 \\ https://shs.hal.science/halshs-00432789 \\ Preprint submitted on 17 Nov 2009
}

HAL is a multi-disciplinary open access archive for the deposit and dissemination of scientific research documents, whether they are published or not. The documents may come from teaching and research institutions in France or abroad, or from public or private research centers.
L'archive ouverte pluridisciplinaire HAL, est destinée au dépôt et à la diffusion de documents scientifiques de niveau recherche, publiés ou non, émanant des établissements d'enseignement et de recherche français ou étrangers, des laboratoires publics ou privés. 
LA GESTION PUBLIQUE DE LA NATURE LITTORALE, EN QUETE DE LEGITIMITE ? L'EXEMPLE DU CONSERVATOIRE DU LITTORAL SUR LES RIVAGES GUYANAIS 


\title{
PUBLIC MANAGEMENT OF COASTAL NATURE LOOKING FOR MORE LEGITIMACY? THE CONSERVATOIRE DU LITTORAL IN THE FRENCH GUYANA
}

\author{
CELine BARTHON, VinCENT ANDREU-BOUSSUT, MOISE TSAYEM-DEMAZE
}

\author{
UMR ESO 6590 CNRS \\ ESO Angers (CARTA), 5bis Boulevard Lavoisier 49045 ANGERS Cédex \\ ESO Le Mans (GREGUM) Avenue O. Messiaen 72085 Le Mans Cédex \\ Contact : celine.barthon@univ-angers.fr, vincent.andreu-boussut@univ-lemans.fr,moise.tsayem-demaze@univ-lemans.fr
}

\begin{abstract}
RESUME
Cet article s'attache à restituer les principaux résultats d'une recherche financée en 2007-2009 dans le cadre d'un programme interdisciplinaire du CNRS dédié à l'ingénierie écologique. Si la démarche s'est intéressée à la gestion de la nature sur les littoraux français, ce texte se focalise sur l'action du Conservatoire du Littoral en Guyane française et fournit ainsi l'occasion d'observer et de comprendre comment un outil d'intervention foncière - une agence publique en charge de la protection des littoraux en métropole comme en outre-mer - conçu et calibré pour le contexte métropolitain dans les années 1960-70 voit ses mobiles et formes d'action perpétuellement adaptés pour répondre aux exigences de contextes locaux très différentes de celles de la métropole. Incarnant depuis sa création en 1975 une réussite incontestable de la part de l'Etat dans la lutte contre l'urbanisation, il reste cependant difficile à évaluer les effets du système inédit d'intervention et de gestion de la nature initié par le Conservatoire. A la fois top-down et bottom-up, ce système propose en amont de négocier les acquisitions avec les élus locaux et en aval d'en confier la gestion et la valorisation aux acteurs locaux, garantissant une légitimité sociale de la protection des espaces acquis. Dans un contexte de retrait de l'Etat, de revendications identitaires et politiques particulièrement affirmées et d'enjeux environnementaux se hissant à l'échelle internationale, l'efficacité et la légitimité d'intervention du Conservatoire sont parfois mises à mal, même si cette dernière reste garantie par la règle juridique. Afin de mettre en œuvre sa stratégie à l'horizon 2050, l'agence publique témoigne d'un grand pragmatisme et d'une réelle capacité à faire perdurer le système, même si les liens avec les acteurs locaux sont loin d'être toujours pérennes. L'analyse systématique des plans de gestion et de valorisation et des discours de l'ensemble des acteurs en présence révèle la construction d'une gouvernance des aires protégées pour lesquelles le projet de protection est fondamental. Pour renforcer la légitimité sociale de la protection de la nature, les sites sont néanmoins systématiquement proposés comme des espaces publics où les pratiques écotouristiques sont fortement mobilisées et promues. Reste à savoir si le modèle de développement proposé participe d'un développement durable du territoire guyanais.
\end{abstract}

MOTS CLE : LITTORAL, ESPACE PROTEGE, GUYANE FRANCAISE, CONSERVATOIRE DU LITTORAL, LEGITIMITE, APPROCHE PARTICIPATIVE, GOUVERNANCE, ECOTOURISME.

\begin{abstract}
First results of a CNRS research program about ecological engineering operation which took place in 2007-2009, this text deals with nature management on the French Guyana coast and focuses on the Conservatoire du Littoral (CL), a public agency in charge of the protection of coastal areas. Since its creation in 1975, the CL has become the first landlord of coastal zones in all the French territories, protecting natural areas and landscapes in most cases from urbanisation. Dealing with local stakeholders to undertake nature management in its properties, the nature management system initiated by the CL is a mix between top-down and bottom-up territorial action. In a context of a falling public authoritary, the Guyana coast gives the opportunity to study how the CL is dealing with local stakeholders when political and cultural issues are very far away from its own 2005-2050 long term strategy initiated in the French metropolis. Even though the CL's legitimacy is defined by the state laws, its action is sometimes made difficult by a dynamic demography, identity struggles, cultural complaints and international scale ecological issues, limiting the social acceptance and actual realisation of nature protection and management. Nevertheless, the public agency keeps re-assessing its legitimacy in the local context, and shows its own ability not only to keep the system working - however difficult the relation with stakeholders may be - but also to build a pragmatic and original project. Indeed, the management and development plan analysis and the stakeholders' discourse database reveal the beginning of a governance under construction. In order to reinforce the social acceptance of nature protection, natural areas are often proposed as public spaces where ecotourism practices are systematically promoted. But is that model ecologically and socially efficient?
\end{abstract}

KEYWORDS: COASTAL ZONE, PROTECTED AREA, FRENCH GUYANA, CONSERVATOIRE DU LITTORAL, LEGITIMACY, PARTICIPATORY APPROACH, GOVERNANCE, ECOTOURISM. 


\section{INTRODUCTION}

Cet article est issu des travaux de recherche menés entre 2007 et 2009 dans le cadre d'un projet portant sur la gestion des usages sociaux des espaces naturels protégés du littoral français métropolitain et guyanais, financé par le programme interdisciplinaire du CNRS « Ingénierie écologique ». Il met l'accent sur la question de la légitimation de l'action publique et du rôle de l'Etat dans les nouveaux dispositifs de gouvernance territoriale en Guyane française, à travers l'observation d'un acteur privilégié et incontournable de la protection de l'espace littoral : le Conservatoire du Littoral (CL).

«Entre patrimonialisation et médiation» (Kalaora et Konitz, 2004), cet établissement public fait en effet figure d'exception sur le territoire français. Qu'il s’agisse de sa politique d'acquisition foncière négociée avec les élus ou encore du choix de déléguer aux acteurs locaux la gestion de ses sites, son fonctionnement, souvent présenté comme un modèle de gouvernance sur le littoral métropolitain, ne peut qu’éveiller la curiosité scientifique. En dehors de la thèse de J. Klein (2003a), aucune recherche n'a d'ailleurs investigué son action sur les rivages de la Guyane française alors même que, comme le soulignent justement Grenand et al. (2006), "l'application de la loi républicaine soulève de nombreuses ambiguïtés et ne va pas de soi » dans ce département français d'outre-mer.

Dès lors, interroger la gestion quotidienne des sites acquis par le Conservatoire et la construction de sa politique d'intervention dans le cadre de sa stratégie nationale à long terme (Conservatoire du Littoral, 2006), permet de questionner les limites de son action et de son efficacité. Dans quelles mesures, le "modèle " métropolitain se trouve-t-il écorné en outre-mer dans un contexte politique et culturel guyanais tendu par le retrait progressif de l'Etat et par des revendications communautaires fortes (notamment vis-à-vis du foncier et du maintien des droits d'usages dans certains espaces) ? De quelle marge de manœuvre l'établissement public dispose-t-il dans ce contexte bien éloigné de la métropole et sous quelles formes et avec quels acteurs locaux se construisent alors les nombreuses opérations de gestion des sites acquis ?

Car évidemment, si la légitimité du Conservatoire à intervenir sur le territoire guyanais est acquise d'un point de vue juridique, au nom du principe de l’identité législative - sous réserve d’adaptations liées aux spécificités de l’outre-mer -, son action de concertation dans la gestion des espaces dont il est propriétaire, qui constitue le cœur de ses missions, semble plus difficile à mener. A l’image de la longue négociation sur la création du Parc amazonien de Guyane, l'essentiel de cette question réside dans la difficulté à asseoir l'acceptation sociale de nouveaux territoires protégés dont les valeurs, les usages et les représentations font l'objet de divergences fortes entre les sociétés locales et les acteurs institutionnels, même décentralisés.

Loin de privilégier une approche uniquement monographique et idiographique, les investigations nous ont conduit dans l'ensemble des sites du Conservatoire en Guyane française mais aussi dans un certain nombre d'entre eux en métropole. La lecture que nous en livrons se trouve donc contextualisée au-delà du seul département guyanais, à une échelle plus vaste, permettant de discerner disparités et similitudes des particularités de fonctionnement du Conservatoire, en fonction des territoires. D’un point de vue méthodologique, les analyses présentées ici sont le fruit d’observations de terrain, de l'examen systématique des plans de gestion et de valorisation des sites et de toute une série d'entretiens menés auprès des représentants locaux du Conservatoire du Littoral à Cayenne, auprès des gestionnaires des sites comme, et plus largement, des acteurs liés à la protection de l'environnement en Guyane ${ }^{1}$. Enjeux écologique et sociétaux sont décryptés en faisant jouer les échelles qui leur donnent le plus de sens possible - échelle nationale / internationale, échelle métropolitaine / guyanaise - afin d'évaluer les variations d'appréciation et de regard sur ce qui « fait patrimoine » ou ne le fait pas par les différents groupes sociaux en présence. Cet article rejoint ainsi l'ensemble des travaux qui ont récemment été publiés sur l'état de la gouvernance dans le cadre de l'intervention du Conservatoire du Littoral en métropole (Narcy et al., 2009 ; Gérard 2008 et 2009) dont il constitue ainsi un prolongement des réflexions.

\section{I - LE CONSERVATOIRE DU LITTORAL EN GUYANE : UN OUTIL METROPOLITAIN DES ANNEES 1970 EN OUTRE-MER}

\section{1 - Un établissement public à la légitimité sociale et scientifique éprouvée sur le terrain}

Destinée à la protection et à la gestion (indirecte) du littoral, la création du CL doit être reliée aux soubresauts de la politique d'aménagement touristique du littoral initié par l'Etat dans les années 1960 en région (cf. Mission Racine en

1 Voir liste des entretiens en bibliographie. 
Languedoc-Roussillon dont le secrétaire général, Pierre Raynaud, sera le premier directeur du Conservatoire). Préconisé par la DATAR (Rapport Piquard en 1973), son objectif premier fut de soustraire (par l'acquisition foncière) à l'urbanisation, un certain nombre de site « naturels » afin de maintenir des coupures vertes qui, à terme, devront constituer le « tiers naturel ou sauvage » d’un littoral métropolitain de plus en plus convoité.

Planificateur et initiateur de modes de gestion décentralisés avant l’heure, tout en gardant la maîtrise foncière et la primauté des choix/orientations de gestion de ces propriétés, le CL s’inscrira néanmoins en rupture avec l'héritage de la Mission Racine en plaçant les collectivités-gestionnaires face à leur responsabilité (Andreu-Boussut, 2008 ; Kalaora et Konitz, 2004). C’est d’ailleurs cette organisation qui lui permettra de tirer sa légitimité sur le terrain puisque les élus sont placés au cœur du dispositif de par leur présence au sein des deux conseils (conseils de rivages et d'administration), les acquisitions étant théoriquement soumises à l’avis des conseils municipaux (Le Pensec, 2001 ; Gérard et Marrou, 2009).

Néanmoins, ses acquisitions vont être guidées par un certain nombre d’objectifs. Priorité sera donnée aux espaces sous pressions, en cours de banalisation ou menacés, dont l'accessibilité est limitée ou pour lesquels, la maîtrise foncière publique est la condition de la pérennité d'activités considérées comme protectrice des milieux et paysages. Dès lors, la légitimité d'intervention du Conservatoire ne peut-être qu'évolutive et réflexive, ces différents critères faisant par définition l’objet d'une certaine subjectivité car variant en fonction du temps et des contextes géographiques.

A l'échelle de la Guyane, trois objectifs sont visés dans la stratégie à long terme 2005-2050 des rivages français d’Amérique (Conservatoire du Littoral, 2006) :

- la préservation des petits archipels côtiers, riches en vestiges historiques, en particulier les îles du Salut et les îles de Rémire. En contribuant à la protection, à l'ouverture et à la mise en valeur du patrimoine de ces îles en partenariat avec le Centre Spatial Guyanais (CSG) et la Direction Régionale des Affaires Culturelles (DRAC), le Conservatoire témoigne ici d'une vision patrimoniale élargie dépassant la notion même de « tiers naturels ».

- la protection et la valorisation de grands espaces naturels littoraux afin de participer à la constitution, aux côtés des réserves naturelles, d’un réseau d'espaces naturels protégés représentatifs de cette partie du continent sud-américain. La mangrove (absente du littoral métropolitain mais commune en Guyane) se retrouve ici au premier plan.

- la sauvegarde des rivages et des monts de Cayenne, dans le cadre de la composition d'une ceinture d'espaces naturels longeant le littoral de la presqu'île de Cayenne, et formant des coupures d'urbanisation.

Pour autant, ces trois objectifs ne sont pas figés. Depuis 2005, date à la quelle la stratégie d'acquisition à été édictée, les " hot-spot » de biodiversité et notamment les savanes menacées par des enjeux économiques (infrastructure et pression agricole) peuvent faire aussi l'objet d'actions de protection, d'inventaire et d'acquisition par le CL (entretien J. Burle).

Ainsi, le patrimoine foncier du Conservatoire est-il amené à évoluer : présent en 2008 sur une dizaine de sites équivalents à 12400 ha - 3400 ha d'espaces terrestres et 9000 ha de mangrove - [cf. tableau 1] la stratégie nationale à long terme en pointe une quinzaine pour une surface totale de 27000 ha, tout en reconnaissant «le caractère peu significatif des surfaces en raison de leur inaccessibilité » (Conservatoire du Littoral, 2006). Dans ces conditions, la question de la protection estelle justifiée ? Car au-delà des surfaces acquises par l'établissement public lui permettant de se rapprocher de l'objectif d'un tiers naturel ${ }^{2}$ se pose la problématique de l'accessibilité d'une nature presque "vierge ", nécessitant à priori peu ou pas de mesures de gestion écologique. Quels partenaires alors solliciter dans un contexte régional où la protection de l'environnement est loin de constituer une priorité ?

\section{2 - Loin de la métropole, des enjeux sociaux et environnementaux propres au littoral guyanais}

Marqué par de grands espaces "beaucoup plus vierges, moins urbanisés et peu accessibles comparés à la métropole » (entretien J. Burle), les rivages guyanais ne représentent que 10\% de la surface totale du territoire. Outre les promontoires rocheux de la presqu'île de Cayenne et des îles, quelques cordons sableux, des zones de marais et de savanes, c'est la mangrove qui domine ce littoral puisqu'elle représente $80 \%$ du linéaire côtier. Le trait de côte y est d'ailleurs extrêmement changeant, la dynamique côtière étant marquée par des cycles d'accumulation sédimentaire (zone sous influence amazonienne) et d’érosion extrêmement rapides et démesurés comparés à la métropole (Prost et al. 2001). L’application de la loi littorale et notamment des 50 pas géométriques est donc peu efficiente, les parcelles octroyées en bord de plage voyant leur superficie fluctuer au gré de ces cycles. Pour autant, le Conservatoire s'est porté gestionnaire des 50 pas géométriques à Cayenne et Rémire-Montjoly (comme lui permet la loi de 1996) en vue de créer un sentier littoral et de limiter la pression urbaine sur cette zone instable.

2 En 2008, le Conservatoire assurait la protection de 11\% du linéaire côtier métropolitain contre 7,4\% du rivage guyanais (MEEDDAT Conservatoire du Littoral, 2008), ce qui, par rapport au début des années 2000, constitue une nette amélioration (Klein J., 2003). 
Tableau 1 : Le patrimoine foncier du Conservatoire du Littoral en Guyane, de l'estuaire du Maroni à celui de l'Oyapok. Source : Conservatoire du Littoral, DIREN, entretiens.

\begin{tabular}{|c|c|c|c|c|c|c|c|}
\hline Nom & Communes & Surface & $\begin{array}{c}\text { Date } \\
\text { d'acquisition }\end{array}$ & Caractéristiques & Usages & $\begin{array}{c}\text { Autres } \\
\text { dispositif } \\
\text { ou } \\
\text { inventaire } \\
\text { présents } \\
\text { sur le site } \\
\end{array}$ & Gestionnaire \\
\hline $\begin{array}{l}\text { Pointe } \\
\text { Isère - } \\
\text { Kanawa }\end{array}$ & $\begin{array}{l}\text { Awala- } \\
\text { Yalimapo, } \\
\text { Mana }\end{array}$ & 1593 ha & 1998 & $\begin{array}{l}\text { Forêt côtière sur } \\
\text { cordon sableux ; } \\
\text { marais et mangroves } \\
\text { sur plaine inondable. }\end{array}$ & $\begin{array}{l}\text { Agriculture sur } \\
\text { abatis, chasse, pêche } \\
\text { et prélèvements } \\
\text { divers. }\end{array}$ & $\begin{array}{l}\text { RNN de } \\
\text { l’Amana, } \\
\text { Zone } \\
\text { Ramsar, } \\
\text { PNRG, } \\
\text { ZDUC }\end{array}$ & $\begin{array}{l}\text { En cours } \\
\text { d'affectation } \\
\text { RN / PNRG } \\
\text { (nouveau } \\
\text { gestionnaire de } \\
\text { la RNN depuis } \\
\text { 2008) }\end{array}$ \\
\hline $\begin{array}{l}\text { Crique et } \\
\text { Pripri de } \\
\text { Yiyi }\end{array}$ & $\begin{array}{l}\text { Sinnamary, } \\
\text { Iracoubo }\end{array}$ & 9859 ha & 1995-1999 & $\begin{array}{l}\text { Zone humide (marais } \\
\text { et mangrove); } \\
\text { savanes et forêts } \\
\text { inondables. }\end{array}$ & $\begin{array}{l}\text { Protection, éducation } \\
\text { à l'environnement, } \\
\text { écotourisme (carbet) } \\
\text { Chasse (interdite) et } \\
\text { pêche (réglementée) }\end{array}$ & $\begin{array}{l}\text { ZNIEFF } \\
\text { (type } 1 \text { et 2) } \\
\text { Zone } \\
\text { Ramsar } \\
(1998)\end{array}$ & $\begin{array}{l}\text { SEPANGUY } \\
\text { Commune de } \\
\text { Sinnamary } \\
\text { (depuis 2008) }\end{array}$ \\
\hline $\begin{array}{l}\text { Iles du } \\
\text { Salut }\end{array}$ & Cayenne & 0,2 ha & 1995 & $\begin{array}{l}\text { Patrimoine bâti : } \\
\text { maison du directeur } \\
\text { du bagne }\end{array}$ & $\begin{array}{l}\text { Muséographique : } \\
\text { musée du bagne. }\end{array}$ & $\begin{array}{l}\text { Propriété du } \\
\text { CNES } \\
\text { Site Inscrit }\end{array}$ & AGAMIS \\
\hline $\begin{array}{l}\text { Iles de } \\
\text { Rémire }\end{array}$ & $\begin{array}{l}\text { Cayenne, } \\
\text { Rémire- } \\
\text { Montjoly }\end{array}$ & 41 ha & 2000 & $\begin{array}{l}\text { îlets rocheux } \\
\text { recouverts d'une forêt } \\
\text { secondaire dense ; } \\
\text { occupation ancienne } \\
\text { par des jésuites ; } \\
\text { bagne. }\end{array}$ & $\begin{array}{l}\text { Ouverture de l'îlet La } \\
\text { Mère aux visites. }\end{array}$ & Site inscrit & $\begin{array}{l}\text { AOT avec la } \\
\text { société } \\
\text { d'excursion } \\
\text { maritime } \\
\text { « Autre Mer ». }\end{array}$ \\
\hline $\begin{array}{l}\text { Pointe de } \\
\text { Buzaré }\end{array}$ & Cayenne & 0,6 ha & $1983-2006$ & $\begin{array}{l}\text { Pointe rocheuse } \\
\text { (intérêt géologique) } \\
\text { plantée de palmiers } \\
\text { formant une } \\
\text { « coupure verte » } \\
\text { dans le centre de } \\
\text { Cayenne } \\
\end{array}$ & $\begin{array}{l}\text { Lieux de détente et de } \\
\text { rassemblement ; } \\
\text { panneaux } \\
\text { d'interprétation ; } \\
\text { observation. }\end{array}$ & & $\begin{array}{l}\text { Commune de } \\
\text { Cayenne }\end{array}$ \\
\hline $\begin{array}{l}\text { Anse et } \\
\text { Mont } \\
\text { Bourda }\end{array}$ & Cayenne & 24 ha & $1995-2006$ & $\begin{array}{l}\text { Colline boisée ; îlot } \\
\text { de forêt secondaire en } \\
\text { zone urbaine } \\
\text { surplombant une côte } \\
\text { rocheuse }\end{array}$ & $\begin{array}{l}\text { Sentier littoral ; lieu } \\
\text { de culte (chemin de } \\
\text { croix et calvaire) }\end{array}$ & $\begin{array}{l}\text { Site Inscrit } \\
\text { ZNIEFF } \\
\text { (type 1) }\end{array}$ & $\begin{array}{l}\text { En cours } \\
\text { d'affectation }\end{array}$ \\
\hline $\begin{array}{l}\text { Montabo } \\
\text { et son anse }\end{array}$ & Cayenne & 11 ha & $1995-2006$ & $\begin{array}{l}\text { Colline boisée en } \\
\text { zone urbaine }\end{array}$ & $\begin{array}{l}\text { Sentier littoral ; } \\
\text { défrichements et } \\
\text { campements (avant } \\
\text { acquisition du CL) }\end{array}$ & $\begin{array}{l}\text { Site inscrit } \\
\text { ZNIEFF } \\
\text { (type 1) }\end{array}$ & $\begin{array}{l}\text { Commune de } \\
\text { Cayenne }\end{array}$ \\
\hline $\begin{array}{l}\text { Salines de } \\
\text { Montjoly }\end{array}$ & $\begin{array}{l}\text { Rémire- } \\
\text { Montjoly }\end{array}$ & 20 ha & $1998-2006$ & $\begin{array}{l}\text { Zone humide (en zone } \\
\text { résidentielle) ou se } \\
\text { côtoient lagunes, } \\
\text { marais et mangroves ; } \\
\text { plage. }\end{array}$ & $\begin{array}{l}\text { Sentier éducatif, } \\
\text { observatoires ; site de } \\
\text { ponte de tortue ; } \\
\text { exutoire de la saline } \\
\text { en saison des pluies } \\
\end{array}$ & $\begin{array}{l}\text { ZNIEFF } \\
\text { (type 1) }\end{array}$ & $\begin{array}{l}\text { Commune de } \\
\text { Rémire } \\
\text { Montjoly } \\
\text { Association } \\
\text { Kwata } \\
\end{array}$ \\
\hline $\begin{array}{l}\text { Mont } \\
\text { Mahury }\end{array}$ & Cayenne & 166 ha & 1998-2006 & $\begin{array}{l}\text { Mont boisé } \\
\text { périurbain ; vestiges } \\
\text { d'occupation } \\
\text { précolombienne et } \\
\text { coloniale. }\end{array}$ & $\begin{array}{l}\text { Sentier; fouille ; } \\
\text { défrichements et } \\
\text { campements (avant } \\
\text { acquisition du CL) }\end{array}$ & $\begin{array}{l}\text { Site inscrit } \\
\text { ZNIEFF } \\
\text { (type 1) }\end{array}$ & $\begin{array}{l}\text { En cours } \\
\text { d'affectation }\end{array}$ \\
\hline $\begin{array}{l}\text { Montagne } \\
\text { d'Argent }\end{array}$ & Ouanary & 740 ha & 1998 & $\begin{array}{l}\text { Presqu'île dans } \\
\text { l'estuaire de } \\
\text { l'Oyapock ; vestiges } \\
\text { d'occupation } \\
\text { précolombienne et } \\
\text { coloniale. }\end{array}$ & & Site inscrit & $\begin{array}{l}\text { En cours } \\
\text { d'affectation }\end{array}$ \\
\hline
\end{tabular}

Légende : PNRG : Parc Naturel Régional de Guyane - ZDU : Zone de Droits d’Usages Collectifs- RNN : Réserve Naturelle Nationale, ZNIEFF : Zone Naturelle d'Intérêt Ecologique, Faunistique et Floristique - SEPANGUY : Société d'Étude, de Protection et d'Aménagement de la Nature en GUYane CNES : Centre National d’Etudes Spatiale - AGAMIS : Association pour Gérer l'Architecture et le Musée des Iles du Salut - AOT : Autorisation d’Occupation Temporaire. 
Sur ces espaces côtiers, les enjeux de protection de la biodiversité sont réels, même si la forêt amazonienne présente sur 90 \% de la surface du département attire tous les regards dans ce domaine, surtout depuis la création du Parc Amazonien (Tsayem-Demaze, 2008a). Ces enjeux s'appuient sur de multiples périmètres de protection telles que les réserves naturelles de l'Amana, de l'île du Grand Connétable ou de Kaw-Roura; des zones humides reconnues par la convention internationale de Ramsar (Basse-Mana, marais de Kaw et estuaire de la Sinnamary), auxquelles s’ajoutent les sites du Conservatoire du Littoral et le Parc Naturel Régional de Guyane. Soulignons cependant que les enjeux de conservation paraissent plus axés sur la présence d'espèces emblématiques comme les tortues marines (luth, verte et olivâtre) qui « monopolisent » à elle seule la majorité des recherches mais aussi des conflits entre conservationnistes et population autochtone (Collomb G., 2009).

Parallèlement à cette situation, l'ensemble des communes littorales rassemble environ 70\% de la population guyanaise (Tsayem-Demaze, 2008b). Pluriethnique et multiculturelle, celle-ci connait une forte croissance depuis les années 1980 qui s’appuie sur une natalité élevée (responsable de 75\% de la croissance) et une immigration difficile à contrôler. Cette dynamique démographique touche surtout la plaine littorale, notamment la presqu'île de Cayenne où la pression foncière et urbaine contribue à accroître la tension sociale et la pression sur l'environnement. De plus si l'on ajoute à ces caractéristiques,

- le régime foncier qui, en Guyane, fait de l'Etat le principal propriétaire à hauteur de $90 \%$ du patrimoine foncier contre $9 \%$ pour les privés, CSG compris, et moins de $1 \%$ pour les collectivités, communes et département- (TsayemDemaze, 2008b ; Elfort, 2002)

- la présence de communautés autochtones Amérindiennes, revendiquant des usages traditionnels et ayant obtenu des zones de droits d'usages collectifs (ZDU) sur la partie ouest du littoral (y compris sur le périmètre de la Réserve Naturelle de l'Amana)

- $\quad$ une tendance à l'occupation illicite liée à la forte poussée démographique et à l'immigration sous la forme de quartiers spontanés (bidonvilles) ou d'abattis et ce, même sur les terrains du CL (route de la plage à Rémire-Monjoly ; MontMahury),

on comprendra aisément que pour le Conservatoire, la Guyane est un cas qui présente des particularités, tant vis-à-vis de la métropole que des autres rivages français d’Amérique.

Aussi les principes de participation / concertation auprès des collectivités pour la planification des acquisitions revêtent-ils ici un caractère limité, la très grande majorité des préemptions s’effectuant par un transfert d'affectation de l’Etat, généralement de l'ONF vers le CL (ou du CSG vers le CL pour les îles du Salut) : «le CL s'adresse aux services fiscaux et dès lors qu'il n'y a pas d'autres demandes [exemple des savanes pour les besoins agricoles] aucun arbitrage n'est nécessaire » (entretien J. Burle). On notera quelques rares exceptions où l'acquisition relève du droit privé dans le cadre d’opportunités pas forcément planifiées dans les périmètres de préemption.

La co-construction des dispositifs de gestion et de valorisation des différents sites avec les acteurs locaux montre les mêmes limites : «Le CL ne peut pas faire les choses [ici] comme il le fait en métropole, notamment dans les rapports avec les collectivités. Il est évident que les collectivités n'ont pas la même disposition à s'investir dans ce genre projet. Nous avons des priorités sensibles que sont l'habitat, l'assainissement, les écoles ... parce qu'on a une démographie qui explose ! (...) Alors vous comprenez bien que cette problématique [la protection de l'environnement] dans un pays de $90000 \mathrm{~km}^{2}$ dont on occupe à peine 10\%, les maires n'en font pas forcément leur cheval de bataille !» (Entretien R. Lucenay). Dans ces conditions, quelle légitimité accorder à l’Etat et quelle politique de concertation mettre en place dans la durée ?

\section{II - UNE REMISE EN CAUSE DE LA PHILOSOPHIE D’ACTION DU CONSERVATOIRE : DE NOUVEAUX OUTILS POUR UNE AUTRE GOUVERNANCE ?}

\section{1- Des plans de gestion incomplets ...}

Alors que le décret du 29 août 2003 introduit l'obligation pour le CL de mettre en place des «plans de gestion (dits « simplifiés » pour les DOM) attachés aux conventions de gestion, afin de traduire ses exigences en termes de conservation et de définir les responsabilités du gestionnaire », cet outil s’avère peu compatible à la situation guyanaise dans les faits. Preuve en est qu'aucun n'est achevé (cas également de la Réserve Naturelle de l’Amana où le CL est présent) ; quant à leur réalisation et application, celles-ci semblent fortement compromises à court terme tant les sensibilités et les cultures locales, auxquelles le CL est particulièrement attentif, y paraissent peu préparées.

Plusieurs facteurs peuvent expliquer cette situation. Pour les responsables des collectivités guyanaises, les enjeux de protection de la biodiversité paraissent comme une figure imposée et ne tiennent pas compte des spécificités locales bien que des Zones de Droits d’Usages aient été concédées en 1987 aux Amérindiens d’Awala-Yalimapo. (Grenand et al., 
2006). La nature identifiée comme un patrimoine par les organismes rattachés à la protection de l'environnement est souvent perçue par les populations comme un territoire oscillant entre le sacré et l’hostile, ou encore assimilée à une simple « ressource à exploiter ». D’ailleurs, la mosaïque de communautés présente sur le littoral confère à la nature des représentations fort différentes qu'il conviendrait certainement de mieux décrypter. Le rivage protégé est donc loin d’être reconnu pour ses aménités comme il peut l'être en métropole, si ce n’est dans le cadre de sa valorisation urbaine (cf. collines boisées fortement prisées des Monts Bourda et Mahury) et touristique (qui reste cependant limitée). Enfin, il convient de souligner que le patrimoine bâti présent sur les sites du Conservatoire témoigne d'un contexte historiquement marqué où les épisodes de la colonisation (habitations) et du bagne (vestiges plus ou moins conservés) s’avèrent difficiles à intégrer par les communautés locales parmi les héritages à transmettre aux générations futures.

Aussi le CL est-il amené à contourner la défaillance des plans de gestion par la mise en place de projets de valorisation (incluant des données liées aux milieux et aux espèces remarquables) afin de fédérer autour de ses propriétés et surtout de trouver LE gestionnaire, qui saura ancrer localement les actions de conservation tout en assurant le relais en termes de médiation auprès des populations locales et des visiteurs.

\section{2- ... aux projets de valorisation plus ou moins concertés :}

On touche ici certainement une des forces du CL sur le terrain dans sa capacité à s’adapter à des situations variées, à financer des études, des équipements, à innover même en matière de valorisation pour rendre visible ses acquisitions et sa politique, mais également une de ces principales faiblesses dans sa quête de légitimité et, dans une certaine mesure, dans sa dépendance vis-à-vis des collectivités. Le CL se trouve donc dans une situation pas forcément confortable de «propriétaire foncier » mais aussi de «porteur de projet » qu'il se doit de construire, négocier et orienter en fonction de ses objectifs, mais aussi de la capacité du/des gestionnaire(s) pressentis à se l'approprier pour le faire vivre dans la durée et ainsi participer à la patrimonialisation de ses sites.

Finalement, l'intervention sur les milieux (lorsqu'elle existe -mais cela vaut également en métropole-) s'insère dans un projet plus global où les aspects économique (développement local, tourisme), social (ouverture et acceptabilité notamment en terme d'usages) et pédagogique (éducation à l'environnement) sont intégrés à différents niveaux au même titre que l'environnement. On retrouve ici les conclusions de l'article de Narcy et al. (2009) sur l'articulation entre "intervention environnementale et développement durable » analysée à partir de six études de cas en métropole : «les sites du CL et le CL lui-même n'offrent pas une vision univoque du développement durable, le CL apparaissant prêt à s'ouvrir à diverses ambitions dans une optique plus expérimentale que normative ».

Néanmoins, le fonctionnement en réseau des acteurs de la protection et de la gestion (CL, bureaux d'études, ONF, scientifiques ...) participe à la construction et à la circulation de savoirs empiriques et de normes guidant de ce fait l'intervention sur les espaces protégés. Sur ce point, le contexte guyanais n’échappe pas à la reproduction de certains modèles métropolitains en termes :

- d'objectifs (calquer le fameux sentier douanier de métropole sur le rivage de Cayenne afin que la population se tourne vers le littoral, le fréquente et se l’approprie) mais également,

- d'infrastructures comme de dispositifs d'accueil et de médiation auprès des publics, pas toujours adaptés à l'environnement (cf. signalétique sur les espèces et notamment l'avifaune présente sur plusieurs sites bien que les conditions de présence et d'observation ne soient par forcément réunies) et encore moins à la diversité des populations locales dont la participation aux dispositifs d’interprétation reste limitée.

Cependant, comme le souligne le représentant local, « en matière de valorisation, localement, il y a peu d'exemples sur lesquels se reporter. Et les exemples ailleurs, sous peu qu'on les connaisse, ne sont pas forcément transposables... Même en Martinique c'est différent ! [pression touristique plus forte et absence de communautés autochtones] 》 (entretien J. Burle). D’où la nécessité d’innover en s’appuyant sur les savoir-faire du CL mais pas seulement ...

\section{III - ENTRE REPRODUCTION ET INNOVATION OU COMMENT ANCRER LOCALEMENT LA VISION ET LES PROJETS DU CONSERVATOIRE}

\section{1- La priorisation de l'écotourisme dans la valorisation des sites}

Si depuis la loi du 27 février 2002, l’ouverture des sites acquis par le Conservatoire au public est mentionnée parmi les missions de l'établissement public, aucun engagement spécifique ni aucune recommandation générale en matière d'éducation à l'environnement, d'équipement ou d'écotourisme ne figurent dans ses dispositions. En effet, ces aspects de 
valorisation sont contractualisés site par site, avec les gestionnaires, « dans la limite de la vocation et de la fragilité de chaque espace ».

Les disparités métropole / Guyane apparaissent ici de manières criantes : alors que l’on réduit l'accès et la fréquentation dans certains sites en métropole, les sites guyanais voient leur accessibilité aménagée ! Cet argument fait d'ailleurs partie de la stratégie d'action de l'établissement public dans ce département d'outre-mer : " on acquiert des terrains pour les préserver mais aussi pour les valoriser et du coup, que cela soit admis par les politiques » (entretien J. Burle).

En se positionnant comme acteur du développement de l'écotourisme, le Conservatoire n’hésite donc pas à investir dans des équipements plus ou moins couteux afin de participer à la (re)connaissance patrimoniale, écologique et économique de ses propriétés : maison de la nature, mirador, observatoires, sentiers pédagogiques, pontons dédiés à la découverte de la mangrove ou des marais, signalétique... L’ingénierie écologique au service de la valorisation produit des marqueurs spatiaux qui facilitent l’identification des espaces protégés mais participent de leur uniformisation.

Photos 1-2-3 : Dispositif d'accueil et équipements des Pripris de Yiyi (Sinnamary) et logotype du Conservatoire du Littoral (4).

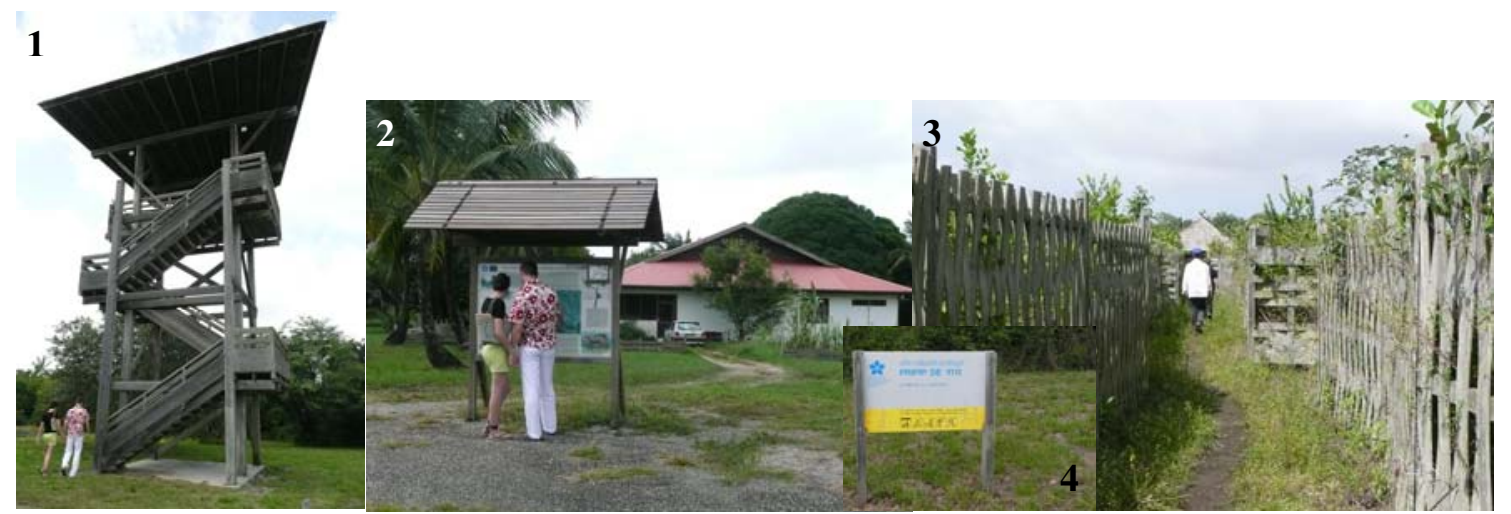

Andreu Boussut V., février 2008.

Par ailleurs, le rôle clé affecté au Conservatoire en faveur de l'émergence d’un écotourisme de qualité ne doit pas occulter un certain nombre de freins liés :

- $\quad$ à la concurrence des pays voisins (Brésil-Pará, Surinam), du Costa Rica, leader sur ce créneau ou encore des autres DOM. Un premier contrat d'études prospectives commandé en Guyane dans le secteur du tourisme en 2005-2006 révèle ainsi que celle-ci est la moins visitée (65 000 visiteurs/an), soit deux fois moins que le Surinam, presque sept fois moins que le Pará et la Réunion, sans compter le Costa Rica qui accueillait en 2004, 1,45 millions de touristes.

- $\quad$ à la cohabitation d'usages diversifiés tels que les activités de prélèvement (chasse, pêche, cueillette) et la culture sur abattis encore fréquentes et même autorisées dans les Zones de Droits d’Usages Collectifs (Pointe Isère-Kanawa). D’ailleurs, il n'existe pas en Guyane de réglementation de la chasse à proprement parler mais simplement des arrêtés ministériels fixant des listes d'espèces protégées, autorisées à la chasse et non à la vente, soit la majorité (DIREN). Le CL fait d'ailleurs pression sur le Préfet afin que celui-ci prenne un arrêté (obtenu en 2008 pour l'îlet La Mère) interdisant le prélèvement d'espèces sur ses sites. Il est à noter que dans les pripris de Yiyi, un «petit» arrêté communal permet d’interdire le port d’arme, sans réelle surveillance ni efficacité relevée (entretien J. Burle).

- à la difficulté de fédérer et d'impliquer les populations locales dans la création d'une offre structurée (Baldy et al. 2000) ce qui se répercute également dans les activités d’hébergements. Nos interlocuteurs ont d’ailleurs fréquemment souligné la quasi mono-activité écotouristique autour des pontes de tortues marines qui conditionne les pics de fréquentation et accroit les tensions tant sur les milieux qu’entre les communautés. Aussi les récents projets développés par le Conservatoire intègrent-ils de plus en plus les dimensions culturelles des espaces, les savoirs locaux et vernaculaires dans la gestion de ses sites (cf. projet en 2008 de valorisation du site de Kanawa situé dans une ZDU à Awala-Yalimapo, incluant un programme de conservation intégré privilégiant gestion collective et valorisation du patrimoine environnemental et culturel de la zone).

Le défi est alors de construire des modes d'intervention qui permettraient de réintroduire ces usages, de mobiliser ces savoirs et plus globalement de créer des partenariats «CL-gestionnaire(s) » autorisant une gouvernance plus participative.

\section{2- Une gouvernance en cours de construction ...}


A partir de l'article de Narcy et al. (2009) dans lequel est esquissé un positionnement des projets métropolitains du CL, et au regard sa capacité à créer des partenariats et à générer un développement durable (en relation avec les territoires qui les supportent), l'action du CL en Guyane se situe essentiellement dans l'ingénierie de projet, afin de dépasser les oppositions et conflits d'usages récurrents entre protection, utilisation et accès à la ressource. En effet, qu'il s'agisse des pripris de Yiyi, des sites de la presqu'île de Cayenne ou plus récemment du site de Kanawa, l'établissement public est à chaque fois à l'origine de la dynamique du projet, de l'acquisition du foncier jusqu'à la conception du programme de valorisation. Dans ce contexte, les partenaires sont donc recherchés en fonction de leur capacité à gérer (ou plutôt ici à entretenir et valoriser) mais aussi à diffuser l'idéal de gestion patrimoniale et intégrée du Conservatoire. Néanmoins il va de soit qu'en Guyane certaines des directives métropolitaines ne sont pas toujours bien reçues, comme nous le confirme le gestionnaire de la Saline de Montjoly. Peu enclin à appliquer les mesures de protection du littoral qu'il considère comme secondaires par rapport aux priorités sociales et économiques de sa commune, il confit être pourtant convaincu que le Conservatoire et son action représentent "une opportunité, tant par rapport à l'éducation qu'au développement durable » (entretien $\mathrm{R}$. Lucenay), les exemples cités mentionnant l'intérêt des supports de médiation de la saline pour l'éducation à l'environnement des jeunes et la création de chantiers d'insertion pour l'entretien des sites. Plus qu'en métropole, les dimensions sociales et économiques de la gestion des milieux soulèvent débat et orientent la dynamique des projets.

Aussi, ces derniers sont-ils amenés à évoluer en fonction de l'intervention (ou le retrait) d'un gestionnaire, tout comme certainement le changement de délégué. Le CL peut alors se positionner comme "co-constructeur d'un idéal et d'un projet » (Narcy et al., 2009) où intérêts et responsabilités entre celui-ci et le(s) gestionnaire(s) sont partagés. L'exemple des Pripris de Yiyi reflète parfaitement cette évolution. Le premier projet de charte de Protection partenariale du bassin versant de la crique Yiyi ${ }^{3}$ élaboré en 1998 n'ayant jamais abouti, le CL se tourne alors vers la commune de Sinnamary qui y assure ainsi l'accueil et l'entretien au début des années 2000. Insatisfaite de ce partenariat, cette dernière se retire en 2004 pour laisser place à la Société d'études, de protection et d'aménagement de la nature en Guyane (SEPANGUY)). Malgré le manque de moyens, cette association réussit à maintenir l'animation du site tout en proposant les bases d'un premier plan de gestion simplifié en 2007 (Conservatoire du Littoral, 2007). Parmi les mesures d'entretien figurent au premier chef la gestion des niveaux d'eau et la mise en place d'un pâturage extensif par un troupeau de buffles pour éviter la fermeture du marais par eutrophisation (signature d'une convention de gestion avec un éleveur de Sinnamary). Durant toute cette période, le Conservatoire n’a pas cessé d'investir dans l'équipement et le fonctionnement du site ${ }^{4}$. Depuis 2008, l'intégration au réseau Ramsar et la mise en œuvre d'un nouveau projet Leader pour le développement rural de la Guyane ont favorisé le retour progressif de la municipalité de Sinnamary dans la gestion du site. La configuration d'acteurs se trouve aujourd'hui élargie (commune, association, éleveur et Conservatoire) à laquelle il faut ajouter le Parc naturel régional de Guyane qui accompagne la stratégie de développement local du Groupe d'action locale (GAL) des savanes (Sinnamary, Iracoubo et Saint-Elie). Dix ans auront donc été nécessaires pour mettre en place les premières bases d'une gouvernance dont les modalités, plus institutionnelles et contractuelles que participatives, soulèvent toutefois la question de la pérennité des projets et des acteurs associés.

Enfin, si le CL se positionne plus rarement comme « suiveur, en situation de veille » (Narcy et al., 2009) en Guyane (sauf peut-être lorsqu'il inscrit son action d'acquisition foncière dans le réseau des Réserves naturelles ou quand il passe une convention avec un acteur privé ${ }^{5}$ ), il reste néanmoins l'appui ou l'intermédiaire indispensable pour initier des partenariats autour de ses acquisitions. Dans le cas des îles du Salut, si l'intégralité de l'archipel est une propriété privée du Centre national d'études spatiales (CNES) ${ }^{6}$, le Conservatoire a néanmoins fait l'acquisition de la maison du directeur de l'ancienne prison afin que l'Association pour gérer l'architecture et le musée des îles du Salut (AGAMIS), rattachée au CNES, puisse élargir ses adhérents et surtout bénéficier de la taxe prélevée par le Conservatoire sur le passage maritime. Au-delà de ces bénéfices pragmatiques, cette association du Conservatoire au devenir des îles du Salut permet au Centre spatial guyanais de revaloriser une image publique mitigée et de trouver, via un établissement public réputé pour sa collaboration avec les collectivités territoriales, de nouveaux partenariats (Klein J., 2003b). La tentative inédite de construire un partenariat avec la commune de Cayenne, alors même que les îles dépendent administrativement de la municipalité, se trouve ainsi grandement facilitée par le capital de confiance dont jouit le Conservatoire auprès des communes, alors même qu'il est un établissement public d'Etat (entretien J. Burle). Bénéficiant d'une image positive et d'un bon tissu de relations avec les

\footnotetext{
3 Projet de charte réunissant le CL, les communes de Sinnamary et d'Iracoubo, le CIRAD (Centre de coopération internationale en recherche agronomique pour le développement) avec le groupement SILVOLAB (Groupement d'intérêt scientifique pour la recherche sur l'écosystème forestier tropical humide en Guyane française) et l’ONF.

4 Réalisation d'un nouvel observatoire ornithologique dans l’Est du marais ; rehaussement des digues de sable pour éviter l'inondation de celles-ci en saison des pluies ; création du seuil de la crique Canceler pour retenir une lame d'eau plus importante dans le marais en saison sèche ; faucardage d'une large zone de pripris ; « refonte » de la maison de la nature en écomusée.

5 Comme c'est le cas pour l'ilet la Mère (Rémire-Montjoly) où la convention de gestion du site, sous la forme d'une Autorisation d'occupation temporaire, a été passée avec un prestataire de transport maritime (le seul en mesure de le faire), afin de rendre accessible l'île tout en suivant un cahier des charges précis mentionnant des restrictions en terme d’usages, d'accès à l’île, de sauvegarde du milieu naturel et d'aménagement.
}

6 Le CNES est présent en Guyane au travers du Centre spatial guyanais (CSG). 
collectivités locales, certaines d'entre elles n’hésitent d'ailleurs pas à le solliciter comme appui à leurs projets, comme c'est le cas de Saint-Elie sur le lac de barrage de Petit-Sault ou encore de Rémire-Montjoly pour l'acquisition de l'habitation jésuite de Loyola. Pourtant, malgré ce climat de relative confiance, le Conservatoire ne reste pas assuré de la gestion des sites, ce qui constitue une limite évidente à cette forme d'association.

\section{CONCLUSION}

Le contexte d'action du Conservatoire du Littoral a bien changé depuis sa création en 1975 en pleine dynamique métropolitaine des Trente Glorieuses. Si pendant les premières décennies, ses interventions ont été fortement marquées par la lutte contre une urbanisation galopante, ses missions ont sérieusement évolué, notamment en termes de service public, et ont connu de ce fait une complexification croissante. Ainsi la capacité du Conservatoire à asseoir sur les sites, au-delà de leur acquisition, l'ouverture systématique au public et plus avant des projets de développement conçus et portés localement est devenue une charge incontournable, même si la loi ne l'y contraint pas. Alors même que le concept de la délégation de gestion des propriétés aux acteurs locaux a constitué un principe d'action particulièrement avant-gardiste, l'interaction avec les acteurs locaux est désormais plus étroite et demande de nombreux «arrangements ", par delà le cadre légal et institutionnel.

L'exemple des rivages guyanais témoigne justement de la manière dont le Conservatoire du Littoral s'acquitte de cette nouvelle autre tâche au cœur de la construction d'une gouvernance de ses aires protégées. Il fournit ici une occasion rare la forêt amazonienne étant beaucoup plus largement investiguée - de comprendre comment l'agence foncière publique se moule dans un contexte environnemental, culturel et politique bien éloigné de celui de la métropole pour proposer des formes renouvelées d'innovation sociale et écologique.

Loin de constituer une administration centralisée, lourde et rigide, le Conservatoire témoigne ici bien au contraire d'une capacité de réflexivité, certainement à relier à sa recherche d'un certain pragmatisme de son action. Bien loin des discours de la stratégie à 2050, ses responsables locaux sont ancrés dans un quotidien suffisamment proche du terrain pour que l'efficacité sociale et écologique, dans le respect des objectifs stratégiques nationaux, prime sur la recherche de résultats tangibles quantitativement. Car l'ancrage territorial de la gestion des sites a longtemps été précaire. En témoignent l'abandon de la gestion des pripris de Yiyi par la commune de Sinnamary en 2003-2004 et la lenteur avec laquelle la commune de Cayenne a récemment signé la convention de gestion de la colline de Montabo. Depuis 20 ans de présence en Guyane, l'histoire de l'antenne locale du Conservatoire a été parfois chaotique, ne disposant d'une équipe pérenne de deux personnes seulement depuis 2006. Pour autant, la panne de gouvernance n'est certainement aujourd'hui qu'un mauvais souvenir car la diversification des gestionnaires (vers les associations et les acteurs privés) et l'élargissement du cercle de partenaires (Office national des Forêts, Parc naturel régional de Guyane...) sont clairement en marche, confirmant la construction progressive d’une gouvernance des aires protégées.

Si le Conservatoire est devenu l'acteur « clé » de la protection du littoral en Guyane et ailleurs en France, grâce notamment aux évolutions législatives récentes (possibilités d’affectation du Domaine public maritime depuis 2002, budget réévalué depuis 2005), un certain nombre de points semblent encore à conforter. La participation des communautés locales dans les projets de valorisation reste ainsi au point mort, même si des avancées se dessinent sur le site de Kanawa à AwalaYalimapo. La question de la viabilité des projets n'est d'ailleurs pas toujours clairement posée : le modèle écotouristique proposé constitue-t-il la voie la plus efficace de développement durable ? Plus largement, le Conservatoire du Littoral se heurte aux mêmes difficultés que le Parc naturel régional de Guyane à faire participer les populations et à trouver des connexions efficaces avec le monde politique. Une solution réside certainement à mieux intégrer en amont usages et représentations locales et multiformes de la nature, tâche complexe nécessitant une connaissance fine des publics et d'autant plus utile que la bonne cohabitation entre les différents usages et usagers semble souvent n’avoir lieu que sur le papier.

\section{BIBLIOGRAPHIE}

Andreu-Boussut V., 2008. La nature et le balnéaire. Le littoral de l'Aude, Ed. L’Harmattan, 356 p.

Baldy B., Madelaine E.., Paulin M-H, 2000. Le challenge de l'écotourisme en Guyane. Antiane-Eco n²46, p.25-28.

Collomb G., 2009. Sous les tortues, la plage ? Protection de la nature et production des territoires en Guyane, Ethnologie française, PUF, p.11-21.

Conservatoire du Littoral, 2006. Conservatoire du littoral - stratégie à long terme 2005-2050, Atlas des espaces naturels protégés du littoral, DVD-ROM, 268 p. 
Conservatoire du Littoral, 2007. Protection du littoral et développement durable: Quels projets? Quels partenaires ? Quelle gouvernance, Atelier du Conservatoire du Littoral, 26-27 sept 2007, 23 p.

Conservatoire du Littoral, 2008, Site de Kanawa à Awala-Yalimapo, Plan de valorisation, ONF, C2R, GADEPAM, 57 p.

DIREN, 2007. Atlas des sites et espaces naturels protégés de Guyane, DIREN Guyane, 96 p.

Elfort M., 2002. De la décentralisation à l'autonomie : la Guyane, Ecole Nationale d'Administration, Revue française d'administration publique, $\mathrm{n}^{\circ} 101$ 2002/1, p. 25-37.

Gérard Y., 2008. Trente ans d’action du Conservatoire du Littoral, Etudes Foncières, n¹31, p. 36-40.

Gérard Y., 2009. Une gouvernance environnementale selon l'Etat ? Le Conservatoire du littoral entre intérêt général et principe de proximité », VertigO - la revue électronique en sciences de l'environnement, Volume $9 n^{\circ} 1$, mai 2009, [En ligne], mis en ligne le 29 mai 2009. URL : http://vertigo.revues.org/index8551.html. Consulté le 09 novembre 2009.

Grenand F. et P., Bahuchet S., 2006. Environnement et sociétés en Guyane française ; des ambiguïtés d'application des lois républicaines, Revue internationale des sciences-sociales, n¹87 2006/1, p.53-62.

Kalaora B., Konitz A., 2004. Le Conservatoire du littoral : entre patrimonialisation et médiation, Annales de géographie ${ }^{\circ}{ }^{\circ 635}$, 2004/1, p. $87-103$

Klein J., 2003 a. Protéger le littoral dans les départements français d'outre-mer, Thèse de doctorat de géographie, Université de Paris 4, 545 p.

Klein J., 2003b. Les petites îles au large des départements français d’Amérique : entre convoitises et protection, Géoconfluences, http://geoconfluences.ens-lsh.fr.

Le Pensec L., 2001. Vers de nouveaux rivages. Rapport à Monsieur le Premier Ministre Lionel Jospin sur la refondation du Conservatoire du Littoral, Paris, 2 vol. 99 et 54 p.

MEEDDAT, Conservatoire du Littoral, 2008. Contrat d'objectifs : rapport de performance 2008, $51 \mathrm{p}$.

Narcy J-B., Michel C., Bouteloup C., 2009. Comment s'articule l'intervention environnementale avec les pratiques et utopies du développement durable? L'exemple du Conservatoire du Littoral et de ses partenaires gestionnaires ", Développement durable et territoires [En ligne], Varia, mis en ligne le 16 juillet 2008, Consulté le 09 novembre $2009 . \quad$ URL : http://developpementdurable.revues.org/index6163.html

Prajoewari N., 2008, Bilan de la réserve de l'Amana en 2007, Réserve naturelle de l’Amana, 10 p.

Prost T.M., Charron C., Caillé O., 2001. Le système de dispersion amazonien : dynamique côtière régionale et locale, impacts sur le littoral guyanais. In Atlas illustré de la Guyane, sous la direction de Barret J., IRD Editions, p. 58-61.

Réserve Naturelle de l'Amana, 2003. Plan de gestion de la réserve naturelle de l'Amana (section A), 81 p.

SEPANGUY, Conservatoire du Littoral, 2007. Plan de gestion simplifié, Pripri de Yiyi / Maison de la nature, Commune de Sinnamary, Guyane française, $67 \mathrm{p}$.

Tsayem-Demaze M., 2008 a. Croissance démograhique, pression foncière et insertion territoriale par les abattis en Guyane française, Norois, 206, 2008/1, p. 111-127.

Tsayem-Demaze M., 2008 b. Le parc amazonien de Guyane française : un exemple du difficile compromis entre protection de la nature et développement, article 416, Cybergéo, Revue Européenne de Géographie, p. 1-23.

\section{Entretiens février 2008}

J. Burle (représentant local du Conservatoire du Littoral à Cayenne) et J. Mariton (chargée de mission), Cayenne.

S. Ferraroli (chargée de mission PNRG - antenne ouest), Mana.

R. Lucenay (directeur des services techniques), mairie de Rémire-Montjoly.

P. Gombault (directeur du PNR Guyane) et L. Garnier (chargé de mission éducation à l'environnement), Cayenne.

A. Chevassu (chef de service Biodiversité) et S. Linares (Atelier Bases de données, SIG-Cartographie et Gestion des Espaces) DIREN Guyane, Cayenne.

Y. Pennec (président de l'association AGAMIS), Centre Spatial Guyanais, Kourou.

M. Delfault, SEPANGUY (animateur gestionnaire du site des pripris de Yiyi et de la Maison de la nature), Sinnamary.

N. Préjoari (gestionnaire de la Réserve naturelle de l’Amana), Awala-Yalimapo. 